\title{
PHYSIOLOGICAL AND BIOCHEMICAL CHANGES FOLLOWING HYPERTHERM TREATMENT*
}

\author{
By JOHN WALLACE, M.B., Ch.B., B.Sc. \\ Capt. R.A.M.C., Army Blood Transfusion Service \\ and S. R. M. BUSHBY, B.Sc. \\ Capt. R.A.M.C., Army Blood Transfusion Service
}

The excellent results which have been claimed in the treatment of resistant cases of gonorrhoea by artificial fever combined with chemotherapeutic measures have caused the increased use of this form of therapy. Treatment which involves the maintenance of the body temperature at $106^{\circ} \mathrm{F}$. for a period of eight hours imposes a severe strain on the vital organs of the body particularly when such treatment is in unskilled hands. The Report of The United States Council on Physical Therapy (1934) shows that twenty-nine deaths occurred among 4,809 patients treated by hyperpyrexia produced by physical agents. The present work has been undertaken because of the high incidence of undesirable features, namely vomiting (31.4 per cent), jaundice (1.4.8 per cent), circulatory collapse (17.7 per cent), incontinence of faeces (5.2 per cent), and deaths $(1.2$ per cent) which occurred among the first 175 cases treated in the hypertherm department of a large venereal diseases hospital.

The object of this work has been to investigate physiological and clinical changes occurring in patients undergoing hypertherm treatment for gonorrhoea ; to ascertain whether additional sulphonamide chemotherapy increases the risks of such treatment ; and to discover thereby any prophylactic or therapeutic measures which will eliminate or minimize the dangers inherent in hypertherm treatment. Particular attention has been paid to the circulatory system and to haematological and biochemical observations, as well as to the mental state.

\section{Methods}

The patients, all volunteers, showed no abnormality on routine examination apart from resistant gonorrhoea. The routine practice in the hypertherm department was to starve the patient from the night before until the completion of treatment. Abundant saline drinks were given throughout treatment, while nurses skilled in fever therapy tended and encouraged. Sedatives were given in order to calm restless patients.

\section{RESULTS OF TREATMENT}

Detailed clinical, haematological and biochemical studies were made on seven patients undergoing the routine treatment (Series A). The following observations were noted.

\section{Haematological and biochemical observations}

(1) There was a transient haemodilution and polymorphonuclear leucocytosis in every case with a return towards the pre-treatment levels within twenty-four hours. (2) A rise in serum bilirubin occurred in every case, progressing to clinical jaundice in three cases within forty-eight hours. Observations pointed to this being hepatic in origin. (3) There was a transient rise in non-protein nitrogen, and a transient fall in the carbon dioxide content of plasma and in plasma chlorides. Blood sugar levels showed no significant change.

\section{Clinical features}

Mental state.-Every case showed a striking mental picture, which varied from drowsiness or stupor to marked restlessness and excitement; the same patient might present both features at different periods of the treatment. This mental state was most marked towards the end of treatment, but was observed in one case within the first hour. Patients became disorientated and appeared to become

\footnotetext{
* From a paper read to the Medical Society for the Study of Veneredl Diseases, 24th July, 1943.
} 
insensitive to heat and pain because some had burns which in a normal subject would most certainly give rise to pain. During the restless phase some subjects became extremely violent, sang bawdy songs and shouted abusive words. It was impossible to reason with patients. This picture was typical of cerebral anoxia.

Cyanosis.-Cyanosis was a constant feature developing in these cases; this always appeared within the first hour and persisted. Usually a marked vasodilatation was present, but in Case 7 which is described below, pallor was a striking feature during the last hour of treatment.

Respiratory rate.-This was usually between 30 and 40 per minute during treatment, but in Case 7 the maximum respiratory rate reached 48 and became very shallow.

Pulse.-The rates varied during treatment from 120 to 160 per minute. The volume was good throughout treatment in every case, but Case 7 showed a weak pulse soon after the termination of treatment.

Blood pressure.-Blood pressure showed a rise in systolic pressure within the first hour ; the diastolic pressure either remained the same or rose slightly so that the pulse pressure was increased. The blood pressure during the latter half of treatment returned to its initial level and was maintained until after treatment, when the pressure usually fell to a low but safe level around 100 millimetres of mercury. In Case 7 however, this fall resulted in a state of circulatory collapse. The postural response of the blood pressure when the patient sat sharply upright was maintained after treatment in every case except Case 7 which showed the following response : before treatment, 140/95-150/100 ; after treatment, 90/70-60/50.

Case 7.-The patient was a healthy young airman, aged twenty-two years. The clinical course was similar to the previous cases, although the breathing was rapid, shallow and reached a maximum rate of 48 per minute. Cyanosis was present : restlessness had been present during the first hour, but the patient settled down to a quieter and drowsy state until about two hours before the end of the eight-hour period, when he again became very restless and was given a sedative. The patient became drowsy again and remained in a listless state until the end of the treatment. It was noted during the last hour of treatment that the patient had become pale but circulatory collapse had not developed.

After removal from the cabinet the patient was very listless, he remained cyanosed, his breathing was rapid and shallow and he vomited a small amount of bile-stained fluid. Halfran-hour after the cessation of treatment the blood pressure was 90/70, the pulse 130 per minute and of poor volume. One hour after treatment the blood pressure was still 90/70, the pulse 136 per minute and feeble while respirations were 40 per minute and shallow. The patient's skin was cold and pale, but there was no sweating. The temperature was falling, but the fall was slower than in other cases. At this stage the foot of the bed was elevated and oxygen in high concentration was administered through a B.L.B. inhalation apparatus.

Two-and-a-half hours after the termination of treatment the blood pressure was $80 / 70$, the pulse 150 per minute and almost imperceptible, the respirations were 36 per minute and very-irregular, the skin was cold, pale, cyanosed and moist. The temperature had fallen to $100^{\circ} \mathrm{F}$. Mentally the patient was drowsy and listless and appeared unaware of what was happening or of the severity of his condition. He complained of nausea and wanted to defaecate; later he became incontinent of faeces.

One litre of plasma was then infused rapidly with careful attention to any signs of developing cardiac failure, but to untoward reactions occurred. The following results of the infusion were observed.

\begin{tabular}{l|c|c|c|c}
\hline & $\begin{array}{c}\text { Haemo- } \\
\text { globin }\end{array}$ & $\begin{array}{c}\text { Haema- } \\
\text { tocrit }\end{array}$ & $\begin{array}{c}\text { Plasma } \\
\text { Proteins } \\
\text { gms. per } \\
\text { 100c.cns }\end{array}$ & $\begin{array}{c}\text { Blood } \\
\text { Pressure }\end{array}$ \\
\hline Before infusion & 112 & 45 & $7 \cdot 3$ & $80 / 70$ \\
\hline $\begin{array}{c}\text { Five minutes } \\
\text { after infusion }\end{array}$ & 110 & $44 \cdot 5$ & $7 \cdot 3$ & $100 / 80$ \\
\hline
\end{tabular}

The administration of 5 per cent carbon dioxide and oxygen in high concentration was continued after the infusion ; the colour improved, the pulse showed a fall in rate and an improvement in volume, while the breathing became regular and slower.

Five hours after the end of treatment the blood pressure was $90 / 65$, the pulse 90 per minute and of good volume, and the respirations, 26 per minute, were regular and deeper. The colour was good and the patient was warmer. This improvement was subsequently maintained. 


\section{PHYSIOLOGICAL AND BIOCHEMICAL CHANGES}

Eleven cases were studied (Series B) with three main modifications in the routine treatment as follows. (1) Oxygen in high concentration was given throughout treatment by means of a nasal B.L.B. mask, and carbon dioxide, 5 per cent, was added to the oxygen whenever the breathing became rapid or shallow. The giving of oxygen and carbon dioxide, 5 per cent, was continued for one hour after treatment. (2) The starvation regime was abolished and the patient was allowed a meal after 6 p.m. on the night before treatment ; 50 grammes of glucose was given at 6 a.m. and tea and toast for breakfast on the morning of treatment ; during treatment glucose, 2 per cent, was administered in the saline drinks so that by encouraging all patients to drink at least 4 litres another 80 grammes of glucose was taken. (3) An additional 8 grammes of sodium chloride was given to the patient by mouth on the day before and on the day after treatment. Sedatives were not administered even to a restless patient.

\section{Haematological and biochemical observations}

The results were similar to those in Series A, the main points being (1) a transient haemodilution and polymorphonuclear leucocytosis ; (2) a transient rise in nonprotein nitrogen ; (3) a fall in carbon dioxide content of plasma during treatment ; (4) less tendency for plasma and urinary chlorides to fall during and after treatment ; (5) a constant rise in serum bilirubin. In two cases clinical icterus was observed, and it is again noteworthy that one patient who showed a high initial bilirubin level and a doubtful clinical jaundice had had a hypertherm session ten days previously. This rise in serum bilirubin was demonstrable within four hours after the start of treatment. The only patient examined on the sixth day after treatment showed a serum bilirubin higher than the initial level.

\section{Clinical features}

Mental state.-The patients were more cooperative than those in Series A; stupor and marked restlessness were not seen apart from one patient who did present marked features of cerebral anoxia ; it is noteworthy that this particular patient refused to use the B.L.B. mask.

Cyanosis.-This was not the prominent feature that it was in the patients of Series A, but it did appear, particularly in those who did not tolerate the mask well.

Respiratory rate.-Fespiration was usually between 30 and 40 per minute during treatment, and any tendency of the breathing to become more rapid and shallow was checked by the addition of carbon dioxide, 5 per cent, to the oxygen.

Pulse rates. - The pulse varied during treatment from 110 to 140 per minute, and the volume was good throughout in every case. The rates in this series were slower than in the original series.

Blood pressure.-The readings were similar to those recorded in the patients in Series A, but no patient developed circulatory collapse. The postural response of the blood pressure when the patient was in the upright sitting position was maintained after treatment in every case.

\section{Additional investigations}

An additional forty-two cases were treated by using continuous oxygen therapy and the extra food and glucose. No special investigations were undertaken but clinical observations were made, particular attention being paid to the circulatory state and to the development of jaundice. Circulatory collapse did not develop in any of the patients in this series, in sharp contrast to the original series. The pulse rates varied on the average throughout treatment between 110 and 136 . Three patients developed manifest clinical jaundice, while three other cases were regarded as showing equivocal clinical jaundice. Vomiting was uncommon, a striking difference from the original series.

\section{Series C}

Nineteen cases were observed under the modified conditions described in Series B with however additional premedication with sulphathiazole, 6 grammes of this drug being given in divided doses of 2 grammes each at 10 p.m., 2 a.m. and 6 a.m. ; treatment in the hypertherm cabinet began at 8 a.m. as usual. The main haemato- 
logical and biochemical results were similar to those of Series B, but the following points are emphasized.

Serum bilirubin:-There was a constant rise in the level as a result of treatment, apart from one patient who was exposed to a temperature of $106^{\circ} \mathrm{F}$. for only one and a half hours and who did not show any increase. In three cases clinical jaundice developed, while three patients had an equivocal clinical jaundice.

Hippuric acid test.-The normal excretion figures for the intravenous test range from 0.7 to 0.95 grammes of benzoic acid. Each of the eight cases examined showed a marked reduction in liver function as judged by the excretion of benzoic acid as hippuric acid.

Effect of abundant chlorides on development of jaundice.-Of the five patients receiving abundant chlorides two developed clinical jaundice and one an equivocal clinical jaundice. On the other hand, in the control series there was one case of doubtful clinical jaundice. The administration of abundant chlorides did not prevent the development of jaundice.

Clinical features.- These were similar to those observed in Series B. Cyanosis and other features of anoxia were not completely abolished by the continuous administration of oxygen and carbon dioxide, but these features were less prominent in this series than in Series A. One case did present marked features of cerebral anoxia, and treatment was términated after only one and a half hours at $106^{\circ} \mathrm{F}$. There was no evidence of the development of circulatory collapse, and the postural response of the blood pressure when the patient was made to sit sharply upright was maintained. Vomiting was uncommon and in no case did it interfere with the oral administration of fluid in sufficiently large quantities to prevent dehydration.

Circulation rate.-As judged by the Decholin arm-to-tongue circulation time, a rapid circulation was shown at various stages during treatment. The most rapid circulation time observed was five seconds and the slowest ten seconds.

Serial electrocardiographic tracings.-These taken before and immediately after treatment showed no evidence of myocardial stress.

Sulphathiazole.-The additional chemotherapy of 6 grammes of sulphathiazole did not appear to produce any haematological, biochemical or clinical changes which had not already been produced by hypertherm treatment alone in the previous series. It seemed unlikely from these observations that such premedication with sulphathiazole in any way increased the hazards of hypertherm treatment.

\section{DISCUSSION OF RESULTS}

The most prominent and constant observations are the features of anoxia and the bilirubinaemia.

Anoxia.-The development of cyanosis and other features of anoxia as a result of exposure to high temperatures has been noted frequently but the cause is uncertain. Hartman (1937) has shown that there is a reduced arterial and venous oxygen and carbon dioxide content of the-blood in hyperpyrexia, and he recommends oxygen therapy in hypertherm treatment. On the other hand, Looney and Borkovic (1942) claim that there is no reduction in the oxygen content of arterial and venous blood in fever treatment, and therefore oxygen therapy is irrational ; these workers have shown however that a significant fall in the mean carbon dioxide levels of both arterial and venous blood occurs. The present results indicate that oxygen and carbon dioxide therapy is of value for the following reasons : (1) improved colour of the patient; (2) slower pulse rate ; (3) improved general condition of the patient ; (4) lesss vomiting ; (5) avoidance of circulatory collapse.

Since continuous oxygen therapy was introduced in the present series over seventy patients have been treated without any evidence of circulatory collapse. The administration of oxygen and carbon dioxide for at least one hour after treatment seems important, because the circulatory collapse commonly occurs then, and in the only case of collapse (Case 7, Series A) studied in detail there was associated with the collapse a marked reduction in the carbon dioxide content of the plasma. 


\section{PHYSIOLOGICAL AND BIOCHÉmiCAL CHANGES}

Cyanosis and other features of anoxia in lesser degree persist however in spite of continuous oxygen therapy. In the absence of any myocardial failure this suggests either a vast increase in oxygen consumption or the presence of a histotoxic type of anoxia due to actual cellular damage by high temperatures. A complete answer to the cause of anoxia will be obtained only after measurements of oxygen consumption and of the oxygen and carbon dioxide content of arterial and venous blood have been taken, studies which have not been possible in the present investigation. The method of administration of oxygen was important. The ordinary type of nasal B.L.B. mask proved unsatisfactory at first because of non-cooperation of the patients. The importance of a preliminary practice with the mask was then realized, and this was carried out on the day before treatment. The nursing staff must pay constant attention to the administration of oxygen and encourage the patient. An oro-nasal mask proved unsatisfactory because of the difficulty in administering fluids and the additional sense of claustrophobia which these patients experience. Another difficulty encountered in the use of the ordinary type of nasal B.L.B. mask was the small capacity of the rebreathing bag. In many patients the tidal air was increased and exceeded the capacity of the bag. This difficulty was overcome by fixing a larger bag to the mask.

Bilirubinaemia. - This has been a constant feature apart from one case in which the patient was exposed to a temperature of $106^{\circ} \mathrm{F}$. for only one and a half hours. The increased pigment appears to be of hepatic origin; there is no alteration in the stool even in the cases of clinical jaundice, and the fall in haemoglobin and red cell count represents a pure dilution because there is rapid recovery to the pre-treatment level without any evidence of a reticulocytosis as in a haemolytic condition. In addition there is marked impairment of hepatic function as judged by the intravenous hippuric acid test. Little attention in the past appears to have been paid to the development of jaundice after hypertherm treatment, and it has been claimed in some American hypertherm clinics (personal communication) that jaundice will never occur if a sufficient amount of chlorides is administered to the patient because jaundice is an index of chloride deficiency. The authors are well aware of the fact that chloride deficiency results from the excessive vomiting of jaundice but they do not know whether deficient chlorides produce jaundice. Addison's disease, which is characterized by chloride deficiency, does not present the symptoms of clinical jaundice. An attempt, however, to eliminate bilirubinaemia by the administration of abundant chlorides was made. This showed that there was no relationship whatever between chloride deficiency and the development of jaundice.

The studies showed that jaundice might develop at any time from twenty-four to ninety-six hours after treatment, and the bilirubinaemia was progressive and persistent after treatment. The cause of the liver damage was not ascertained. Continuous oxygen therapy did not prevent the incidence or lessen the degree of bilirubinaemia. At the same time continuous oxygen therapy did not prevent the development of anoxia and therefore the liver damage might be due to anoxia or to the direct effect of high temperatures on the liver cells. The importance of such liver damage lies in the possibility of development of chronic liver damage, particularly in the case requiring repeated treatment, for example gonococcal arthritis. Prevention of liver damage of the above types has been attempted in Series $B$ and in Series $C$ by abolishing the previous starvation routine of the hypertherm department and giving additional glucose before treatment (Dunlop, Davidson and McNee, 1943). The failure of this measure in preventing liver damage may have been due to inadequate dosage, and in future much larger amounts of glucose will be given for two days before and for two days after treatment. The effect of additional protein in preventing liver damage as recommended by Whipple (1942) has not been tested, but that this may be worth while in future investigations is suggested by two observations in the present series. The fall in plasma protein concentration is greater than can be explained by pure dilution in some cases and recovery of this plasma protein level is slower than recovery of haemoglobin and red cell counts to the pre-treatment level. It is 
also interesting that the only patient (Case 7) who received additional protein in the form of plasma intravenously did not show a marked rise in serum bilirubin in spite of severe anoxia and circulatory collapse. In cases in which repeated treatments are required it is recommended that the serum bilirubin level at least should be estimated at the outset, and if possible an intravenous hippuric acid test should be performed before further hypertherm treatment is undertaken.

Circulatory collapse.-The striking difference between the original Series A on the one hand and Series B and C on the other is the complete absence of circulatory collapse in the two latter series. This great improvement appears to be associated with the introduction of continuous oxygen therapy. There are three main possible reasons for the development of circulatory collapse : (1) reduction in circulating blood volume as in dehydration (Nadal, Pedersen and Maddock, 1941) ; (2) myocardial failure ; (3) failure of the respiratory and vasomotor centres.

There has been no evidence of a reduction in the circulating blood volume in any of these patients, in fact haemodilution has been frequently observed. Increase in blood volume probably occurs to compensate for the increase in the vascular bed associated with marked vasodilatation. Haemoconcentration, which is a feature of circulatory collapse in dehydration (Nadal, Pedersen and Maddock) and is described as a constant feature of hypertherm treatment (Neymann, 1938), has never been observed in the present series. Haemoconcentration, if it does develop, is probably a warning sign that the patient is becoming dehydrated. It has been possible in the present series to administer sufficient fluid and salt by mouth to prevent dehydration, weight loss and the circulatory collapse due to dehydration.

There has not been any evidence of myocardial failure in the cases studied. Venous pressure appears to be raised in most cases from an early stage of the treatment, but this rise is associated with the vasodilatation and not with other features of cardiac failure. Electrocardiographic tracings do not show any evidence of myocardial damage and the circulation time is rapid. Further, the patient in Case 7 who developed circulatory collapse showed no ill effects as a result of rapid infusion of plasma which would produce circulatory overloading in a patient with circulatory collapse due to myocardial failure.

The absence of circulatory collapse since continuous oxygen therapy was introduced, particularly oxygen combined with carbon dioxide given at intervals during and after treatment, suggested that the circulatory collapse previously seen was a failure of respiratory and vasomotor centres. Case 7 showing circulatory collapse had a very marked reduction in carbon dioxide content of plasma associated with the collapse. The improvement in the condition of the patient concerned started with the rapid infusion of plasma which caused a transient rise in the circulating blood volume above normal and a rise in the blood pressure. Such infused plasma in an individual who has not suffered an acute reduction in the circulating blood volume, is rapidly lost from the circulation (Sharpey-Schafer and Wallace, 1942). However the clinical improvement was maintained by the administration of carbon dioxide in addition to oxygen. Particular attention was paid to the rate and depth of breathing in these patients, and when a patient breathing pure oxygen showed shallow rapid breathing; carbon dioxide, 5 per cent, and.oxygen was substituted for pure oxygen, with marked improvement in respiratory function.

Renal impairment.-Most patients show a small transient rise of non-protein nitrogen which disappears within three days in every case. Urinary output was well maintained; there has been no evidence of any abnormalities in the urine except the presence of bile pigments in those cases in which jaundice has developed. There is therefore no evidence of gross renal damage in the present series, but the Report of the Council on Physical Therapy records albuminuria as a complication of hyperpyrexia. More detailed studies on renal function following hypertherm treatment are required because the kidneys are particularly sensitive to lack of oxygen (Tomb, 1941). The danger of renal damage from hyperpyrexia is greatest in cases with pre-existing renal impairment. Blatt, Fouts and Page (1938) have shown that heat produced by electromagnetic induction slightly reduces the urea and creatinine clearances in subjects with renal disease. It has been 


\section{PHYSIOLOGICAL AND BIOCHEMICAL CHANGES}

similarly noted that renal impairment following a haemorrhage of one litre of blood in man is noted only in subjects with pre-existing renal disease (Wallace and Sharpey-Schafer, 1941).

Haematological changes.-The finding of a transient polymorphonuclear leucocytosis as a result of hyperpyrexia has been noted frequently (Simon, 1936 ; Neymann, 1938; Hargraves and Doan, 1939). The significance of this response is uncertain. The transient haemodilution which has been observed probably represents an increase in blood volume to compensate for the increased vascular bed in vasodilatation. Such an increase in blood volume has been recorded by Bazett (1938), who has shown that the blood volumes of healthy men in Philadelphia are from 15 to 40 per cent higher in summer than in winter.

Delayed reactions.- The possibility and danger of permanent damage to the liver, particularly as the result of repeated treatment at short intervals, has been emphasized. Some patients are listless or depressed for two or three days after treatment but in the cases studied to date no permanent damage to the nervous system appears to have resulted from the anoxia or high temperatures. The danger of production of cerebral lesions by anoxia has been noted particularly in carbon monoxide poisoning (Price, 1941), which in rare instances results in severe amnesia and aphasia which may not appear until several weeks after the poisoning. A clinical picture indistinguishable from that of hysteria and taking many months to clear up is more commonly seen in carbon monoxide poisoning. That nervous lesions do actually result from hyperpyrexia has been noted. Stein (1936) describes bilateral pyramidal tract signs as an unusual complication of treatment by hyperthermy, while Stecher and Solomon (1936) report on the development of facial paralysis and aphasia during the treatment of gonococcal arthritis with artificial fever.

Indications for terminating treatment.-The treatment has considerable dangers, and it is desirable to reduce the length of treatment as far as is compatible with therapeutic results. The duration of treatment must be decided by the medical officer observing each case, but the following are suggested as indications for terminating treatment immediately, in addition to a fall of systolic blood pressure below 100 millimetres of mercury.

(1) Disorientation, for example failure to appreciate surroundings or time, or obvious inability to cooperate because of cerebral anoxia. (2) Pulse rate persistently over 160 per minute. (3) Respiration rate persistently over 50 per minute. (4) Temperature of $107^{\circ} \mathrm{F}$. for a period of a quarter of an hour. (5) Restlessness and violence. (6) Coma. (7) Pallor superimposed on cyanosis. (8) Intolerance on part of patient ; the restlessness of an intolerant patient adds greatly to the dangers inherent in the treatment. (9) Catarrhal infection and nasal obstruction; these would be regarded as contra-indications to treatment. (10) Persistent vomiting.

Pre-hypertherm and post-hypertherm treatment. - Hypertherm treatment must be regarded as similar to a surgical operation of election, and there should be careful pre-hypertherm and post-hypertherm treatment. The following recommendations are the salient features of such treatment.

Pre-hypertherm treatment.-(1) A thorough rolitine clinical examination by a medical specialist to exclude patients with cardiovascular, respiratory, renal or hepatic disease. (2) A careful explanation of the treatment should be given by the medical officer beforehand to prepare the patient for the physical and mental ordeal of prolonged exposure to a high temperature. (3) Obviously unsound psychological types should be avoided or, if such an individual does accept treatment and becomes difficult, treatment should be terminated immediately. (4) Patients should have a liberal diet with large amounts of carbohydrate and possibly protein. (5) Patients should practise using a B.L.B. mask.

Post-hypertherm treatment.-(1) The use of oxygen and carbon dioxide immediately after treatment for at least one hour or until colour improves. (2) Patients should be treated as cases of acute hepatitis and kept warm in bed for at least three days or until jaundice has cleared. (3) If a subsequent hypertherm session is considered, liver function should be investigated.

\section{SUMMARY}

(1) Two hundred and fifty-four cases undergoing hypertherm treatment at $106^{\circ} \mathrm{F}$. 


\section{THE BRITISH JOURNAL OF VENEREAL DISEASES}

for eight hours have been studied clinically. Thirty-seven of these cases have been the subject of a detailed clinical, haematological and biochemical investigation.

(2) The most constant and prominent features have been the development of anoxia and bilirubinaemia, progressing to manifest clinical jaundice in thirty-seven cases. Hippuric acid tests show a considerable reduction in liver function.

(3) Continuous oxygen and carbon dioxide therapy lessens but does not abolish the features of anoxia. The administration of oxygen and carbon dioxide does appear however to prevent the development of circulatory collapse. The prevention of liver damage is discussed. Vomiting has become less frequent since oxygen therapy was introduced.

(4) This complication of circulatory collapse is a failure of the vasomotor and respiratory centres and is not due to a reduction in the circulating blood volume or to myocardial failure. Morphine is absolutely contra-indicated.

(5) There is a transient polymorphonuclear leucocytosis and a transient haemodilution.

(6) There is a small transient rise in non-protein nitrogen and a tendency for plasma chlorides and urinary chlorides to fall.

(7) Indications for terminating treatment and recommendations for prehypertherm and post-hypertherm treatment are formulated.

(8) Sulphathiazole premedication with 6 grammes of the drug does not increase the hazards of this treatment.

Our thanks are due to Maj.Gen. L. T. Poole, D.S.O., M.C., Director of Pathology, for permission to publish this paper and for the encouragement given to us throughout, and to Brig. L. E. H. Whitby, C.V.O., M.C., for his keen interest and advice. Our thanks are also due to Col. J. A. Stirling for facilities to work in the hospital which he commands; to Lt.-col. A. J. King, Officer in charge of the Hypertherm Department, for permission to study cases ; to Maj. C. R. Lane and Maj. H. Bathurst Norman, for assistance with the laboratory work and electrocardiographic tracings ; and to Q.M.S. Blake, Army Blood Supply Dapot, for his enthusiastic support.

\section{REFERENCES}

Bazett, H. C. (1938) Proc. XVI Int. physiol. Congr. Zurich, 2, 76.

Blatt, E., Fouts, P. J., and Page, I. H. (1938) Amer. J. med. Sci., 196, 340.

Dunlop, D. M., Davidson, L. S. P., and McNee, J. W. (1943) Text Book of Medical Treatment, 2nd ed., Edinburgh, p. 201.

Hargraves, M. M., and Doan, C. A. (1939) Proc. Soc. exp. Biol., N.Y., 42, 361.

Hartman, F. W. (1937) J. Amer. med. Ass., 109, 2116.

Looney, J. M., and Borkovic, E. J. (1942) Amer. J. Physiol., 136, 177.

Nadal, J. W., Pedersen, S., and Maddock, W. G. (1941) J. clin. Invest., 20, 691.

Neymann, C. A. (1938) Artificial Fever produced by Physical Means, London.

Price, F. W. (1941) Text Book of the Practice of Medicine, 6th ed., London, p. 1355.

Report of Council on Physical Therapy (1934) J. Amer. med. Ass., 103, 1308.

Sharpey-Schafer, E. P., and Wallace, J. (1942) Lancet, 1, 699.

Simon, J. F. (1936) J. Lab. clin. Med., 21, 400.

Stecher, R. M., and Solomon, W. M. (1936) Amer. J. med. Sci., 192, 497.

Stein, I. D. (1936) Arch. phys. Ther., 17, 419.

Tomb, J. W. (1941) Med. J. Aust., 2, 569.

Wallace, J., and Sharpey-Schafer, E. P. (1941) Lancet, 2, 393.

Whipple, G. H. (1942) Amer. J. med. Sci., 203, 477.

\section{DISCUSSION ON THE PRECEDING PAPERS}

Lt.-col. C. M. Spooner, R.A.M.C., said that pyretotherapy had been commenced at No. 1 Canadian General Hospital about fifteen months previously. All the patients treated suffered from persistent and chemo-resistant urethritis with gonococci present in the secretion. In addition a number of cases of gonorrho:al arthritis and of gonorrh seal proctitis had been treated. Three hundred and six fever sessions had been given to 176 patients. He had looked up the records of 100 consecutive cases of gonorrho:al urethritis to compare with Lt.-col. King's figures. In sixty-five of the 100 cases a satisfactory eight-hour fever session was accomplished. In thirty-five cases the fever session was not completed. Of the sixty-five patients receiving an adequate fever session fifty-seven were completely cured; in six cases a second session was required and there were two cases classified as complete failures. The number of days in hospital prior to hyperthermy averaged forty-nine; the pre-pyrexial chemotherapy with a sulphonamide averaged 82.5 grammes ; one patient had received a total of 333.5 grammes of sulphonamide previous to pyrexial treatment. In thirty-five cases they were unable to give a satisfactory session of fever 